\title{
Movement in High School: Proportion of Chinese Adolescents Meeting 24-Hour Movement Guidelines
}

\author{
Li Ying ${ }^{1}$, Xihe Zhu ${ }^{2}{ }^{1}$, Justin Haegele ${ }^{2}$ and Yang Wen ${ }^{3, *}$ \\ 1 School of Sport and Physical Education, Huainan Normal University, Huainan 232038, Anhui Prov, China; \\ y10608@hnnu.edu.cn \\ 2 Department of Human Movement Sciences, Old Dominion University, Norfolk, VA 23508, USA; \\ x2zhu@odu.edu (X.Z.); jhaegele@odu.edu (J.H.) \\ 3 Center of Jiangsu Sports Health Engineering Collaborative Innovation, Nanjing Sport Institute, \\ Nanjing 210014, Jiangsu Prov, China \\ * Correspondence: 2001070012@nsi.edu.cn
}

Received: 6 March 2020; Accepted: 28 March 2020; Published: 1 April 2020

check for updates

\begin{abstract}
The purposes of this study were (a) to examine the proportions of adolescents in China who partially or fully meet three 24-h movement guidelines on physical activity, screen-time, and sleep duration and (b) to examine whether there were gender differences in the proportion of boys and girls meeting these guidelines. The sample was made up of high school adolescents from an eastern province of China $(N=1338)$. The participants completed a self-reported survey on demographic variables and weekly health behaviors including physical activity, screen-time, and sleep duration. A frequency analysis was conducted to summarize the number of 24-h movement guidelines met of the total sample and by gender; chi-squared tests were used to examine the gender differences in the proportion of students meeting different guidelines, independently and jointly. A high proportion of adolescents did not meet physical activity $(97.2 \%, 95 \% \mathrm{CI}=96.2-98.0 \%)$, or sleep $(92.1 \%, 95 \%$ $\mathrm{CI}=90.6-93.5 \%)$ guidelines, but met screen-time $(93.6 \%, 95 \% \mathrm{CI}=92.4-94.7 \%)$ guidelines. Overall, only $0.3 \%(95 \% \mathrm{CI}=0.1-0.6 \%)$ of the sample met all three guidelines, $8.8 \%(95 \% \mathrm{CI}=7.5-10.2 \%)$ met two, $85.8 \% \%(95 \% \mathrm{CI}=84.0-87.4 \%)$ met one, and $5.1 \%(95 \% \mathrm{CI}=4.0-6.4 \%)$ met none. There was no statistically significant percentage difference between female and male participants in meeting physical activity, screen-time viewing, or sleep duration guidelines, independently or jointly ( $p$ values $>0.05$ ). These figures of participants meeting all three guidelines or physical activity and sleep independently are much lower than many estimates in prior research internationally. Considerations to improve adherence to physical activity and sleep guidelines are critical in this population.
\end{abstract}

Keywords: adolescence; gender; screen time; sleep; physical activity; prevalence

\section{Introduction}

Pediatric obesity is often cited as a major and pressing health concern $[1,2]$ that can lead to numerous physiological (e.g., high blood pressure, type 2 diabetes) and psychological (e.g., depression, poor quality of life, low self-esteem) issues throughout the lifespan [3-5]. Consistent with international trends [1], national data has demonstrated that China has experienced an alarming increase in pediatric obesity in the past 20 years, as well as associated physiological conditions (i.e., hypertension, diabetes) [6,7]. According to Wang and colleagues [6], this steady increase in obesity in China has coincided with the country's rapid economic growth, and may be a result of multiple lifestyle factors, including unhealthy diet, lack of physical activity (PA), and increased sedentary behavior. With this increase in pediatric obesity, estimates in 2013 suggested that approximately $23 \%$ of boys and $14 \%$ of 
girls in China were overweight or obese [1], which is comparable to many Western countries. Of further concern, China is projected to have the highest number of obese children in the world by 2030 [8,9].

Given the deleterious effects associated with pediatric obesity, close monitoring of children's weight and the promotion of healthy lifestyle behaviors are of concern in China [6,10]. PA, sedentary behavior, and sleep duration are codependent lifestyle behaviors that together account for the 24-h daily cycle [11,12] and may be of interest with regard to reducing pediatric obesity [13-18]. Reflecting the belief that the whole day matters, recent years have seen the development and adoption of movement-related recommendations across the 24-h period [11-13]. For example, the 24-h movement framework presented by Tremblay and colleagues [11] includes recommendations for adolescents aged 14-17 years to engage in (a) at least $60 \mathrm{~min}$ of moderate-to-vigorous PA, (b) two or less hours of recreational screen time, and (c) 8 to $10 \mathrm{~h}$ of sleep per night. Central to the integrated nature of the 24-h movement framework paradigm is the concept that meeting all three movement behavior recommendations may have a greater association with beneficial health outcomes, such as reducing the risk of pediatric obesity, than meeting a single guideline [12]. Supporting this assertion, an emerging body of evidence suggests that meeting all three 24 - $h$ movement guidelines is associated with lower rates of overweight and obesity [13-18], as well as other favorable physiological $[16,17]$ and psychological outcomes [5] during childhood and adolescence.

The emergence of this more holistic and comprehensive perspective of health behaviors, which supports the integrated benefits of meeting all three 24 -h movement guidelines, has prompted research in a number of countries that examines the proportions of youth who meet these guidelines $[13,19]$. For example, a recent cross-sectional study [12] using nationally represented data from the 2016-2017 National Survey of Children's Health in the US demonstrated that just $9.4 \%$ of youth aged 10-17 years met all three 24-h movement guidelines, whereas nearly the same amount $(9.1 \%)$ met none of these guidelines. Similarly, compliance with 24 -h movement guidelines was recently reported to be alarmingly low among adolescents in Hong Kong, where just $1.0 \%$ of a sample of 692 adolescents (aged 11-17 years) met all three guidelines. The current body of evidence suggests that just a small percentage of youth in these studies are experiencing the benefits associated with meeting each of the three 24-h movement guidelines [13-18].

Given issues concerning pediatric obesity in China [6,7], research examining adherence to the 24-h movement guidelines may be timely. However, few studies have examined adherence to 24-h movement guidelines in China [7,13,20]. For example, Guan and colleagues [7] examined the proportion of children (aged 4.1 to 6.3 years) from three kindergartens in Beijing meeting movement guidelines suggested by the World Health Organization, and reported that just $15.0 \%$ met all three guidelines, and $2.7 \%$ did not meet any guidelines. The current research in this area of inquiry in China has focused on pre-school [7] or elementary-aged children [13,21], and no studies, to the knowledge of the authors, have examined proportions of adolescents meeting these health-related guidelines in China. Adolescence may be a particularly important age to examine, given that health-related behaviors become more volitional and less dictated by parents as children grow older $[11,22]$ and the risk for pediatric obesity rises with age $[1,23]$. Thus, the primary purpose of this observational, cross-sectional analysis was to examine the proportions of adolescents in an eastern province in China who partially or fully meet the PA, screen-time, and sleep duration guidelines.

Sociodemographic variables may influence the types of activities that youth choose, as well as barriers they experience when engaging in health-related behaviors. Gender, in particular, may be of particular relevance, given that several studies examining movement guideline adherence among children in China [22], as well as other contexts [12,19], have demonstrated that a lower proportion of girls than boys tend to meet these recommendations together and in isolation. This may be an important point in China, where evidence suggests that girls are at a higher risk of experiencing pediatric obesity than boys $[10,24]$. As such, a secondary purpose of this analysis was to examine whether gender differences emerged in the proportion of boys and girls meeting these 24-h movement guidelines. 


\section{Methods}

\subsection{Study Design and Sample}

This observational study collected data from a convenience sample of adolescents in a large regional high school in an eastern province of China. The total high school-aged adolescent population in the school was approximately 4500. Data were collected through self-reported survey in the fall semester (November-December) of 2019. The response rate was 35.9\%, and 1618 students consented and participated in the data collection. The corresponding author's institutional review board (Human Subject Research Ethics Committee) and the local school approved the study protocols. Parental consent was sought for those who were under 18 years of age, and participatory assent was obtained for all participants prior to data collection.

\subsection{Measures and Procedures}

\subsubsection{Demographics}

Adolescents' age, ethnicity, gender, and parents' highest education were collected in the self-reported survey. Specifically, adolescent age was collected as chronological age. Ethnicity included options of Han and minorities, gender included options of male and female, and parent education included options of (a) high school or less, (b) some college or bachelor's degree, and (c) graduate or professional degree.

\subsubsection{4-h Movement Behaviors}

Three questions were utilized to evaluate if participants met the 24-h movement guidelines. These items were modelled after the National Survey of Children's Health from the United States Census Bureau (2019). To assess PA, adolescents were asked: “During the past week, on how many days did you exercise, play a sport, or participate in PA (that resulted in elevated heart rate, accelerated breathing, and/or sweating) for at least $60 \mathrm{~min}$ ?" Eight response options were available ranging from " 0 " to "7 days." A dichotomous variable, aligned with Tremblay and colleagues recommendations [11], was created where responses of "7 days" were coded as "meeting PA guidelines," and all other responses were coded as "not meeting PA guidelines." For screen-time, adolescents were asked: "On an average weekday, besides doing homework, about how many hours do you usually spend watching TV, online videos, browsing internet, or playing video games?" A dichotomous variable was then created where responses of " $2 \mathrm{~h}$ or less" were categorized as meeting 24 -h movement guidelines, and "more than $2 \mathrm{~h}^{\prime \prime}$ as not meeting 24-h movement guidelines. To assess sleep, an open-ended question asked: "During the past week, how many hours of sleep did you have on an average weeknight?" Responses within the range of 8-10 $\mathrm{h}$ for 14-17 years olds [11], and 7-9 $\mathrm{h}$ for 18-19 year olds [25] were coded as "meeting sleep guidelines," and all other responses were coded as "not meeting sleep guidelines."

\subsection{Data Analysis}

During the data screening, participants with missing or invalid question responses $(n=280)$ were listwise removed. As such, the final sample subjects included in the data analysis were only the ones with complete responses $(N=1338)$. We analyzed the data in two steps: A frequency analysis was conducted to summarize the participants demographics and the number of 24-h movement guidelines met of the total sample and by gender. The bias-corrected and accelerated bootstrap 95\% confidence interval (CI) was estimated based on 1000 bootstrap sample [26]. Next, for cells with sufficient sample counts $(n>5)$, we used chi-squared tests to compare the differences in the proportion of meeting different 24-h movement guidelines, independently and jointly, between female and male participants [27]. The data analyses were conducted using SPSS (Ver. 25, IBM; Armonk, NY, USA). Statistical significance was held at the 0.05 level. 


\section{Results}

The sample was gender-balanced, consisting of $49.4 \%(95 \% \mathrm{CI}=46.9 \%-52.0 \%)$ females, but included predominantly Han ethnics $97.5 \%(95 \% \mathrm{CI}=96.7 \%-98.3 \%)$, which was consistent with the local population. On average, the participants were 16.67 years old $(\mathrm{SD}=0.68)$, ranging from 14 to 19 years old. As seen in Table 1, about half of the participants had parents with some college $(47.8 \%)$ or graduate degrees (7.0\%). A high proportion of adolescents did not meet the PA $(97.2 \%, 95 \% \mathrm{CI}=96.2 \%-98.0 \%)$ or age-appropriate sleep $(92.1 \%, 95 \% \mathrm{CI}=90.6 \%-93.5 \%)$ guidelines, but met the screen-time viewing $(93.6 \%$, $95 \% \mathrm{CI}=92.4 \%-94.7 \%)$ guideline. Overall, only $0.3 \%(95 \% \mathrm{CI}=0.1 \%-0.6 \%)$ of the sample met all three of the 24-h movement guidelines, $8.8 \%$ (95\% CI $=7.5 \%-10.2 \%)$ met two, $85.8 \% \%(95 \% \mathrm{CI}=84.0 \%-87.4 \%)$ met one, and $5.1 \%(95 \% \mathrm{CI}=4.0 \%-6.4 \%)$ met none (Table 1 and Figure 1$)$.

Table 1. Demographic characteristics of adolescents in high school.

\begin{tabular}{|c|c|c|c|}
\hline Age Range & $\begin{array}{c}14-19 \text { Years Old } \\
n=1338\end{array}$ & $\begin{array}{l}\text { Female } \\
n=661\end{array}$ & $\begin{array}{c}\text { Male } \\
n=677\end{array}$ \\
\hline Age (y), mean (SD) & $16.67(0.68)$ & $16.62(0.69)$ & $16.71(0.68)$ \\
\hline \multicolumn{4}{|l|}{ Gender $(\%, 95 \% \mathrm{CI})$} \\
\hline Female & $49.4 \%(46.9-52.0 \%)$ & - & - \\
\hline Male & $50.6 \%(48.1-53.0 \%)$ & - & - \\
\hline \multicolumn{4}{|l|}{ Ethnicity $(\%, 95 \% \mathrm{CI})$} \\
\hline Han & $97.5 \%(96.7-98.3 \%)$ & $97.0 \%(95.8-98.0 \%)$ & $98.1 \%(97.2-99.0 \%)$ \\
\hline Minority & $2.5 \%(1.8-3.1 \%)$ & $3.0 \%(2.0-4.2 \%)$ & $1.9 \%(1.2-2.7 \%)$ \\
\hline \multicolumn{4}{|l|}{ Parent Education $(\%, 95 \% \mathrm{CI})$} \\
\hline High school or less & $45.2 \%(42.8-47.6 \%)$ & $42.8 \%(39.0-46.6 \%)$ & $47.6 \%(43.9-51.3 \%)$ \\
\hline Some college or bachelor's degree & $47.8 \%(45.3-50.3 \%)$ & $50.5 \%(46.8-54.3 \%)$ & $45.1 \%(41.5-48.6 \%)$ \\
\hline Graduate or professional degree & $7.0 \%(5.6-8.4 \%)$ & $6.7 \%(4.8-8.6 \%)$ & $7.4 \%(5.8-9.2 \%)$ \\
\hline \multicolumn{4}{|l|}{ PA participation $(\%, 95 \% \mathrm{CI})$} \\
\hline Less than $60 \mathrm{~min} /$ day & $97.2 \%(96.2-98.0 \%)$ & $97.1 \%(95.9-98.2 \%)$ & $97.2 \%(96.0-98.2 \%)$ \\
\hline $60 \mathrm{~min}$ or more/day & $2.8 \%(2.2-3.6 \%)$ & $2.9 \%(2.0-3.9 \%)$ & $2.8 \%(1.8-4.0 \%)$ \\
\hline \multicolumn{4}{|l|}{ Average weeknight sleep $(\%, 95 \%$ CI $)$} \\
\hline Below age-appropriate hours & $92.1 \%(90.6-93.5 \%)$ & $93.2 \%(91.5-94.9 \%)$ & $91.0 \%(88.8-93.1 \%)$ \\
\hline Met age-appropriate hours & $7.9 \%(6.7-9.3 \%)$ & $6.8 \%(5.0-8.8 \%)$ & $9.0 \%(7.1-10.9 \%)$ \\
\hline \multicolumn{4}{|l|}{ Screen-time viewing $(\%, 95 \% \mathrm{CI})$} \\
\hline $2 \mathrm{~h}$ or less/day & $93.6 \%(92.4-94.7 \%)$ & $94.4 \%(92.7-96.1 \%)$ & $92.8 \%(90.7-94.8 \%)$ \\
\hline More than $2 \mathrm{~h} /$ day & $6.4 \%(5.2-7.7 \%)$ & $5.6 \%(4.1-7.1 \%)$ & $7.2 \%(5.5-8.9 \%)$ \\
\hline \multicolumn{4}{|l|}{ 24-h movement guideline $(\%, 95 \% \mathrm{CI})$} \\
\hline Met 0 guideline & $5.1 \%(4.0-6.4 \%)$ & $4.7 \%(3.3-6.2 \%)$ & $5.5 \%(4.0-6.9 \%)$ \\
\hline Met 1 guideline & $85.8 \%(84.0-87.4 \%)$ & $86.8 \%(84.6-89.0 \%)$ & $84.8 \%(82.0-87.3 \%)$ \\
\hline Met 2 guidelines & $8.8 \%(7.5-10.2 \%)$ & $8.2 \%(6.4-10.0 \%)$ & $9.5 \%(7.4-11.7 \%)$ \\
\hline Met 3 guidelines & $0.3 \%(0.1-0.6 \%)$ & $0.3 \%(0.0-0.8 \%)$ & $0.3 \%(0.0-0.7 \%)$ \\
\hline
\end{tabular}

CI: confidence interval; SD: Standard deviation. 


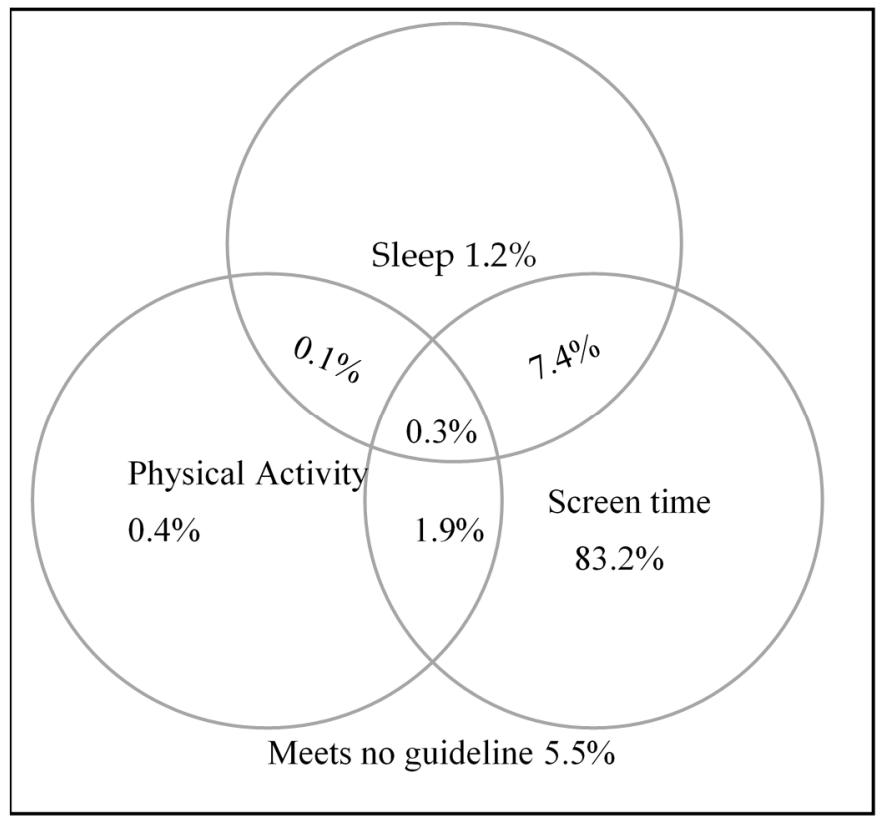

(a)

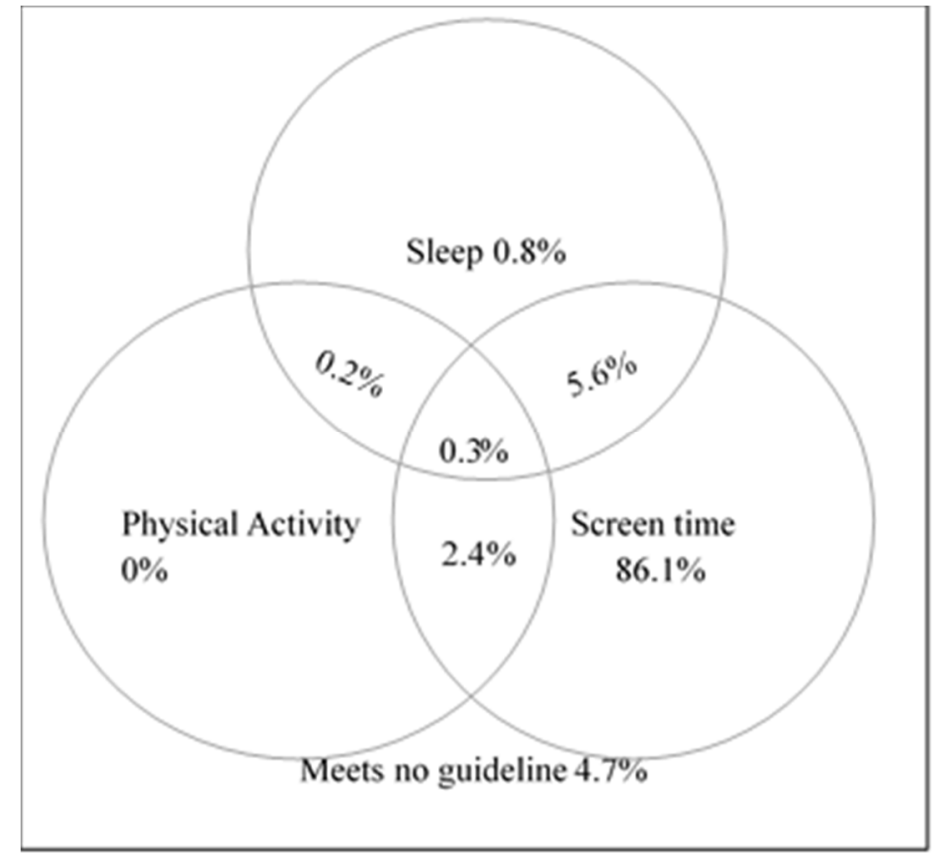

(b)

Figure 1. Percentages meeting 24-h movement guidelines among male (a) and female (b) adolescents. 
There was no statistically significant percentage difference between female and male participants in meeting PA $\left(\Delta \%=0.1 \%, \chi^{2}=0.01, p=0.91\right)$, screen-time viewing $\left(\Delta \%=1.6 \%, \chi^{2}=1.43, p=0.23\right)$, or age-appropriate sleep hour guidelines $\left(\Delta \%=2.2 \%, \chi^{2}=2.20, p=0.14\right)$, independently. As shown in Figure 1, when examining the combination of these guidelines met, for those cells with sufficient subject counts $(n>5)$, we did not find statistically significant percentage differences between female and male participants in meeting sleep and screen-time viewing guidelines jointly $\left(\Delta \%=1.8 \%, \chi^{2}=1.78\right.$, $p=0.18)$, or screen-time viewing and PA jointly $\left(\Delta \%=0.5 \%, \chi^{2}=0.40, p=0.53\right)$. Similarly, there was no significant difference between female and male participants in meeting 0,1 , or 2 guidelines as shown in Table 1 (all $p$ values $>0.05$ ).

\section{Discussion}

The unique contribution of this study is the analysis of the proportions of adolescents in China who individually and jointly adhered to the $24-h$ movement guidelines. Prior research supports the assertion that the whole day matters [11], where the synergistic effects of adherence to all three 24-h movement behavior guidelines outweighs the benefits of meeting guidelines in isolation [11,13-18]. This includes findings focusing on pediatric obesity, where adolescents who meet all three guidelines are at a lower risk of experiencing pediatric obesity than those who meet zero, one, or two [13-18]. Of concern, few adolescents $(0.3 \%)$ adhered to all three 24 -h movement guidelines in this study. This figure appears much lower than many estimates in prior research internationally, which tend to range from $7.2 \%$ [13] to $18.4 \%$ [18]. As such, most adolescents in this study $(99.7 \%)$ did not meet all three $24-\mathrm{h}$ movement guidelines and therefore may not receive the collective, integrative physiological and psychological benefits associated with meeting these guidelines [5,11,13-18].

Of the three 24-h movement guidelines, it is clear that considerations to improve adherence to two of them, PA and sleep, are critical. Alarmingly, just $2.8 \%$ and $7.9 \%$ of the participants met the PA and sleep guidelines, respectively. Given the high percentage of adolescents meeting the screen-time viewing guideline $(93.6 \%)$, these two guidelines are gateways for adolescents to enjoy the integrative benefits of meeting all three 24-h movement guidelines. It is of little surprise that adherence to PA guidelines was the lowest among the three health-related guidelines, given that this is a common trend in research among children in China [13] as well as adolescents abroad [12]. One potential explanation for why adherence to PA recommendations may be low in China is related to academic burden or stress. Academic burden or stress has been identified as a factor that plays a role in reducing the likelihood of adolescents meeting PA guidelines in countries with rigorous academic standards, like China [28,29]. For example, in a cross-sectional analysis of 48,118 school-aged children in Shanghai [29], academic burden was cited as the primary reason for youth not engaging in enough PA, and those who did not report academic burden were about five times more likely to meet PA recommendations. Specifically, high school adolescents on average spent more than $2 \mathrm{~h}$ per day on academic homework [29], although the amount of homework time that was also screen time is not clear. As such, assisting youth in finding a balance between time spent engaged in schoolwork (e.g., homework, studying) and PA time may be one strategy for enhancing PA recommendation adherence among Chinese adolescents.

From a 24-h movement behavior perspective, it could be argued that meeting PA guidelines is linked to meeting sleep guidelines. For example, recent cross-sectional data have demonstrated that youth who regularly meet sleep duration guidelines are also more physically active and have lower rates of pediatric obesity [30,31]. Thus, the benefits associated with meeting sleep guidelines extend beyond simply influencing health-related outcomes, but also can contribute to adherence to other movement behaviors. The low adherence to the sleep guideline in this study was surprising, given adherence to meeting sleep guidelines tends to exceed $40 \%$ in studies internationally $[12,13,15,16]$. The high academic burden during high school years, which has been shown to impact PA participation and screen-time viewing among youth in China [29], might be related to their sleep duration as well.

Recommendations to enhance adherence to the sleep guideline, which would also be relevant for each of the 24-h movement guidelines, may adopt the 5 A's Behavior Change Model recommended by 
Dosh and colleagues [32]. The 5 A's Behavior Change Model includes asking potentially interested youth about the behaviors, advising youth and parents on the importance of the behaviors, assessing potential barriers, assisting with overcoming those barriers with best practices, and arranging for follow-up assessments, if needed. Whereas this model has been used in a variety of contexts to enhance adherence to healthy behaviors [32,33], we are unaware of its adoption, thus far, to enhance adherence to 24-h movement guidelines in totality.

Examining the influence of gender on adherence to the 24-h movement guidelines can help identify if one group needs specialized, focused interventions to enhance adherence. In this study, no significant difference was found between the percentages of girls and boys with regard to meeting each of the guidelines singularly or concurrently. This finding is somewhat surprising, as it conflicts with research examining young children in China [7,24], as well as adolescents in other contexts [14], where boys are more likely to meet 24 -h movement guidelines. It appears that, perhaps, factors influencing adherence to the 24-h movement guidelines among adolescents in this particular high school context (e.g., academic burden) may influence movement behaviors among boys and girls in similar ways. Therefore, these findings suggest that the need for intervention to enhance PA and sleep duration guideline adherence is not gender dependent, and therefore efforts to reduce barriers to health-related behaviors should be provided for both groups.

This analysis has several strengths, including the use of a large sample of adolescents in an understudied geographic area and the adoption of a current movement framework to understand health behaviors. However, limitations are also evident. The primary limitation of this study may be the utilization of self-report measures, rather than objective measures, to measure PA, screen-time, and sleep. While self-report instruments have historically acted as a main source of information on how individual's spend time, recent suggestions to move away from self-report measures cite limitations with the level of detail needed to examine movement across the 24 -h period, as well as concerns related to the influence that social desirability can have on responses where participants may over-report desirable behaviors such as physical activity [34,35]. However, the utilization of self-report may have been advantageous to gain access to a group of individuals in an economically efficient manner that would be challenging otherwise. In addition, just one question was used for each 24-h movement behavior, making it challenging to evaluate the reliability of responses, and the way in which responses were dichotomized may limit variability and represent a narrow viewpoint on what PA, screen-time, and sleep participation are. Finally, while the sample size is relatively large for this study, it is nevertheless a convenient sample, representing an area of an eastern province in China. As such, interpretations of the study findings may be limited to the specific geographic region.

\section{Conclusions}

In conclusion, while over $90 \%$ of the adolescents in this study met the screen-time viewing guideline of $2 \mathrm{~h}$ or less, over $90 \%$ of them did not meet the PA guideline of $60 \mathrm{~min}$ per day, or age-appropriate hours of sleep (8-10 h for 14-17 year olds, 7-9 h for 18-19 years olds). Consequently, less than $1 \%$ of the participants met all three, less than $10 \%$ met two, and about $85 \%$ met one of the 24 -h movement guidelines. About $5 \%$ of the participants met none of the guidelines. Additionally, contrary to some existing reports, we did not find statistically significant difference in the percentage of adolescents meeting these guidelines in their singular or joint status, between female and male participants.

Author Contributions: Conceptualization, X.Z., J.H., and L.Y.; Data curation, Y.W.; Formal analysis, X.Z.; Funding acquisition, Y.W.; Project administration, L.Y. and Y.W.; Writing-original draft, X.Z. and J.H.; Writing一review and editing, L.Y., X.Z., J.H., and Y.W. All authors have read and agreed to the published version of the manuscript.

Funding: This research was in part supported by the Chinese National Funding of Social Sciences (No. 19BTY018).

Acknowledgments: The researchers wish to thank the high school students for their participation in this study and thank the graduate assistants for their timely data entry. 
Conflicts of Interest: The authors declare no conflict of interest. The funder had no role in the design of the study; in the collection, analyses, or interpretation of data; in the writing of the manuscript, or in the decision to publish the results.

\section{References}

1. Ng, M.; Fleming, T.; Robinson, M.; Thomson, B.; Graetz, N.; Margono, C.; Mullany, E.C.; Biryukov, S.; Abbafati, C.; Abera, S.F.; et al. Global, regional, and national prevalence of overweight and obesity in children and adults during 1980-2013: A systematic analysis for the global burden of disease study 2013. Lancet 2014, 384, 766-781. [CrossRef]

2. Swinburn, B.A.; Sacks, G.; Hall, K.D.; McPherson, K.; Finegood, D.T.; Moodie, M.L.; Gortmaker, S.L. The global obesity pandemic: Shaped by global drivers and local environments. Lancet 2011, 378, 804-814. [CrossRef]

3. Davis, A.M.; Bennett, K.J.; Befort, C.; Nollen, N. Obesity and related health behaviors among urban and rural children in the United States: Data from the national health and nutrition examination survey 2003-2004 and 2005-2006. J. Pediatr. Psychol. 2011, 36, 669-676. [CrossRef]

4. Morrison, K.M.; Shin, S.; Tarnopolsky, M.; Taylor, V.H. Association of depression \& health related quality of life with body composition in children and youth with obesity. J. Affect. Disord. 2015, 172, 18-23.

5. Zhu, X.; Haegele, J.A.; Healy, S. Movement and mental health: Behavior correlates of anxiety and depression among children of 6-17 years old in the U.S. Ment. Health Phys. Act. 2019, 16, 60-65. [CrossRef]

6. Wang, Y.; Wang, L.; Qu, W. New national data show alarming increase in obesity and noncommunicable chronic diseases in China. Eur. J. Clin. Nutr. 2017, 71, 149-150. [CrossRef]

7. Guan, H.; Zhang, Z.; Wang, B.; Okely, A.D.; Tong, M.; Wu, J.; Zhang, T. Proportion of kindergarten children meeting the WHO guidelines on physical activity, sedentary behavior and sleep and associations with adiposity in urban Beijing. BMC Pediatr. 2020, 20, 70. [CrossRef] [PubMed]

8. Wang, Y.; Xue, H.; Sun, M.; Zhu, X.; Zhao, L.; Yang, Y. Prevention and control of obesity in China. Lancet Glob. Health 2019, 7, e1166-e1167. [CrossRef]

9. World Obesity Federation. Global atlas on childhood obesity. 2019. Available online: https://www. worldobesity.org/nlsegmentation/global-atlas-on-childhood-obesity (accessed on 3 March 2020).

10. Zhu, X.; Haegele, J.A.; Tang, Y.; Wu, X. Prevalence and demographic correlates of overweight, physical activity, and screen time among school-aged children in urban China: The Shanghai study. Asia Pac. J. Public Health 2018, 30, 118-127. [CrossRef]

11. Tremblay, M.S.; Carson, V.; Chaput, J.P.; Connor, S.; Dinh, T.; Duggan, M.; Faulkner, G.; Gray, C.E.; Gruber, R.; Janson, K.; et al. Canadian 24-h movement guidelines for children and youth: An integration of physical activity, sedentary behavior, and sleep. Appl. Physiol. Nutr. Metab. 2016, 41, S311-S327. [CrossRef]

12. Zhu, X.; Healy, S.; Haegele, J.A.; Patterson, F. Twenty-four-hour movement guidelines and body weight in youth. J. Pediatr. 2020, 218, 204-209. [CrossRef] [PubMed]

13. Roman-Vinas, B.; Chaput, J.P.; Katzmarzyk, P.T.; Fogelholm, M.; Lambert, E.V.; Maher, C.; Maia, J.; Olds, T.; Onywera, V.; Sarmiento, O.L.; et al. Proportion of children meeting recommendations for 24-h movement guidelines and associations with adiposity in a 12-country study. Int. J. Behav. Nutr. Phys. Act. 2016, 13, 123. [CrossRef] [PubMed]

14. Laurson, K.R.; Lee, J.A.; Eisenmann, J.C. The cumulative impact of physical activity, sleep duration, and television time on adolescent obesity: 2011 Youth Risk Behavior Study. J. Phys. Act. Health 2015, 12, 355-360. [CrossRef] [PubMed]

15. Laurson, K.R.; Lee, J.A.; Gentile, D.A.; Walsh, D.A.; Eisenmann, J.C. Concurrent associations between physical activity, screen time, and sleep duration with childhood obesity. ISRN Obes. 2014, 2014. [CrossRef] [PubMed]

16. Carson, V.; Chaput, J.-P.; Janssen, T.; Tremblay, M.S. Health associations with meeting new 24-h movement guidelines for Canadian children and youth. Prev. Med. 2017, 95, 7-13. [CrossRef]

17. Chastin, S.F.; Palarea-Albaladejo, J.; Dontje, M.L.; Skelton, D.A. Combined effects of time spent in physical activity, sedentary behaviors and sleep on obesity and cardio-metabolic health markers: A novel compositional data analysis approach. PLoS One 2015, 10, e0139984. [CrossRef] 
18. Berglind, D.; Ljung, R.; Tynelius, P.; Brooke, H.L. Cross-sectional and prospective associations of meeting 24-h movement guidelines with overweight and obesity in preschool children. Pediatr. Obes. 2018, 13, 442-449. [CrossRef]

19. Knell, G.; Durand, C.P.; Kohl III, H.W.; Wu, I.H.; Gabriel, K.P. Prevalence and likelihood of meeting sleep, physical activity, and screen-time guidelines among US youth. JAMA 2019, 173, 387-389. [CrossRef]

20. Shi, Y.; Huang, Y.H.; Sit, C.; Wong, S.H. Compliance with 24-h movement guidelines in Hong Kong adolescents: Associations with weight status. J. Phys. Act. Health 2020, 17, 287-292. [CrossRef]

21. Thivel, D.; Tremblay, M.S.; Katzmarzyk, P.T.; Fogelholm, M.; Hu, G.; Maher, C.; Maia, J.; Olds, T.; Sarmiento, O.L.; Standage, M.; et al. Associations between meeting combinations of 24-h movement recommendations and dietary patterns in children: A 12-country study. Prev. Med. 2019, 118, 159-165. [CrossRef]

22. Chin, S.T.; Liu, Y.; Hong, J.T.; Tang, Y.; Cao, Z.B.; Zhuang, J.; Zhu, Z.; Chen, P.J. Co-existence of physical activity and sedentary behavior among children and adolescents in Shanghai, China: Do gender and age matter? BMC. Public Health 2018, 18, 1287. [CrossRef] [PubMed]

23. Skinner, A.C.; Ravanbakht, S.N.; Skelton, J.A.; Perrin, E.M.; Armstrong, S.C. Prevalence of obesity and severe obesity in US children, 1999-2016. Pediatrics 2018, 141, e20173459. [CrossRef] [PubMed]

24. Cai, Y.; Zhu, X.; Wu, X. Overweight, obesity, and screen-time viewing among Chinese school-aged children: National prevalence estimates from the 2016 Physical Activity and Fitness in China - The Youth Study. J. Sport. Health Sci. 2017, 6, 404-409. [CrossRef] [PubMed]

25. Hirshkowitz, M.; Whiton, K.; Albert, S.M.; Alessi, C.; Bruni, O.; DonCarlos, L.; Hazen, N.; Herman, J.; Katz, E.S.; Kheirandish-Gozal, L.; et al. National Sleep Foundation's sleep time duration recommendations: Methodology and results summary. Sleep. Health 2015, 1, 40-43. [CrossRef] [PubMed]

26. Shao, J.; Tu, D. The Jackknife and Bootstrap; Springer: New York, NY, USA, 1995.

27. Campbell, I. Chi-squared and Fisher-Irwin tests of two-by-two tables with small sample recommendations. Stat. Med. 2007, 26, 3661-3675. [CrossRef] [PubMed]

28. Zhang, J.; Middlestadt, S.E.; Ji, C.-Y. Psychosocial factors underlying physical activity. Int. J. Behav. Nutr. Phys. Act. 2007, 4, 1-10. [CrossRef] [PubMed]

29. Zhu, X.; Haegele, J.A.; Tang, Y.; Wu, X. Physical activity and sedentary behaviors of urban Chinese children: Grade level prevalence and academic burden associations. Biomed. Res. Int. 2017, 2017. [CrossRef]

30. Kim, Y.; Umeda, M.; Lochbaum, M.; Stegemeier, S. Physical activity, screen-based sedentary behavior, and sleep duration in adolescents: Youth Risk Behavior Survey, 2011-2013. Prev. Chronic. Dis. 2016, 13, E131. [CrossRef]

31. Miller, M.A.; Kruisbrink, M.; Wallace, J.; Chen, J.; Gappuccio, F.P. Sleep duration and incidence of obesity in infants, children, and adolescents: A systematic review and meta-analysis of prospective studies. Sleep 2018, 41, 1-19. [CrossRef]

32. Dosh, S.A.; Holtrop, J.S.; Torres, T.; Arnold, A.K.; Baumann, J.; White, L.L. Changing organizational constructs into functional tools: An assessment of the 5 A's in primary care practices. Ann. Fam. Med. 2005, 3, S50-S52. [CrossRef]

33. Sherson, E.A.; Sherson, E.A.; Yakes Jimenez, E.; Katalanos, N. A review of the use of the 5 A's model for weight loss counseling: Differences between physician practice and patient demand. Fam. Pract. 2014, 31, 389-398. [CrossRef] [PubMed]

34. Rosenberger, M.E.; Fulton, J.E.; Buman, M.P.; Troiano, R.P.; Grandner, M.A.; Buchner, D.M.; Haskell, W.L. The 24-h activity cycle: A new paradigm for physical activity. Med. Sci. Sport. Exer. 2019, 51, 454-464. [CrossRef] [PubMed]

35. Adams, S.A.; Matthews, C.E.; Ebbeling, C.B.; Moore, C.G.; Cunningham, J.E.; Fulton, J.; Herbert, J.R. The effect of social desirability and social approval on self-reports of physical activity. Am. J. Epidemiol. 2005, 161, 389-398. [CrossRef] [PubMed]

(C) 2020 by the authors. Licensee MDPI, Basel, Switzerland. This article is an open access article distributed under the terms and conditions of the Creative Commons Attribution (CC BY) license (http://creativecommons.org/licenses/by/4.0/). 